\title{
DOI: 10.3901/JME.2013.18.038
}

\section{FGH95 镍基粉末高温合金的研究和展望*}

\author{
国为民 ${ }^{1}$ 赵明汉 $^{1}$ 董建新 $^{2}$ 曾 强 $^{1}$ 张龙飞 $^{1}$ 燕 $^{1}$ \\ (1. 钢铁研究总院高温材料研究所 北京 100081 ; \\ 2. 北京科技大学材料科学与工程学院 北京 100083)
}

\begin{abstract}
摘要: 粉末高温合金由于自身特有的组织和性能特点成为先进航空发动机浴轮盘和涡轮挡环的优选材料。总结我国 20 世纪 80 年代初开始研制的第一代镍基粉末高温合金 FGH95 在制粉工艺、粉处理工艺、成形工艺和热处理工艺等方面的研究工作。 着重介绍 FGH95 合金生产中所采用的等离子旋转电极制粉工艺(Plasma rotating electrode process, PREP), 笛分和静电分离去 除夹杂工艺, 直接热等静压(Hot isostatic pressing, HIP)和热等静压十包套锻造(HIP+Forging)成形工艺, 以及固熔+盐淬热处理 工艺。展望氩气雾化制粉(Argon atomizatio, AA)和直接热等静压成形在 FGH95 合金低成本制造上的发展优势和高固溶缓冷热 处理工艺对提高合金抗疲劳裂纹扩展能力的影响, 提出下一步的研究方向。
\end{abstract}

关键词: FGH95 镍基粉末高温合金 制备工艺 组织性能

中图分类号: TF124

\section{Research and Development in FGH95 P/M Nickel Based Superalloy}

\author{
GUO Weimin $^{1}$ ZHAO Minghan ${ }^{1} \quad$ DONG Jianxin $^{2} \quad$ ZENG Qiang $^{1}$ \\ ZHANG Longfei $^{1} \quad$ YAN Ping $^{1}$
}

(1. High Temperature Material Research Institute, Central Iron and Steel Research Institute, Beijing 100081;

2. School of Material Science and Engineering, University of Science and Technology Beijing, Beijing 100083)

\begin{abstract}
Powder metallurgy(P/M) superalloys are selected as turbine disks and turbine ring materials for advanced aero engine applications due to their specific microstructure and high temperature properties. The research on sieve processing, powder treatment processing, case forging processing and heat treatment processing of FGH95 first generation PM superalloy in China during the early stage of 1980 s is reviewed. Various fabrication processes, including plasma rotating electrode process(PREP), sieving, static electricity separating, hot isostatic pressing(HIP) and case forging, solution and salt bath etc, are emphasized. It is also introduced the developing advantages of FGH95 alloy produced using argon atomization(AA) and HIP forging processing, which is low-cost and improvement in the crack propagation resistance due to high temperature solution and slow cooling processing. The future investigation is also discussed.
\end{abstract}

Key words: FGH95 P/M Ni-base superalloy Fabrication processing Microstructure and property

\section{0 前言}

镍基高温合金具有很好的高温性能, 在燃气涡 轮发动机的涡轮部分得到了广泛应用。20 世纪 60 年代末，出现了用粉末治金方法生产高温合金结构 件的新技术，由于粉末高温合金有减少偏析、更有 效的合金化、改善材料的力学性能和降低加工成本

* 国家自然科学基金资助项目(50974044)。20130415 收到初稿, 20130716 收到修改稿
等优点, 因此, 30 多年来得到了迅速发展 ${ }^{[1-3]}$ 。国 外一些较成熟的粉末高温合金如美国的 IN100, Rene95, Rene88DT 等已成为 GE37, F404, CFM56, EJ200 和 PW5000 等先进军民用航空发动机涡轮盘、 轴等关键部件的最佳制造材料, 俄罗斯的 ЭП741НП, ЭП962П 等也是米格 29, 米格 31, 伊 尔 96 , 伊尔 114 等飞机发动机涡轮盘的制造材料, 其中美国 GE 公司的 Rene 95 合金是在 $650{ }^{\circ} \mathrm{C}$ 使用 条件下强度水平最高的粉末高温合金, 表 1 是 Rene 95 与其他合金的性能对比结果 ${ }^{[4]}$ 。 


\begin{tabular}{lcc} 
表 $\mathbf{1}$ & 涡轮盘件用高温合金 $\mathbf{6 5 0}$ & ${ }^{\circ} \mathrm{C}$ 抗拉强度和屈服强度 \\
\hline \multicolumn{1}{c}{ 合金 } & 抗拉强度/MPa & 屈服强度/MPa \\
\hline 热等静压 Rene95 & 1550 & 1200 \\
In-100 & 1350 & 1000 \\
热等静压 Astroloy & 1253 & 913 \\
Waspoloy & 1100 & 850 \\
In-718 & 1105 & 1000 \\
DA718 & 1216 & 1050 \\
\hline
\end{tabular}

FGH95 是我国 20 世纪 80 年代初开始定型研制 的第一个粉末高温合金, 是一种高合金化 $\gamma^{\prime}$ 相沉淀 强化型镍基合金，其成分类似于美国 GE 公司的 Rene95 合金, 其 $\gamma^{\prime}$ 体积分数为 $50 \% \sim 55 \%, \gamma^{\prime}$ 形成 元素质量分数为 $12.8 \%$, 在 $650{ }^{\circ} \mathrm{C}$ 下具有较好的强 度水平, 可用来制造高推比新型发动机的高、低压 浴轮盘, 压气机盘, 浴轮挡环等高温结构件。

\section{FGH95 合金粉末的研究}

\section{1 制粉工艺的研究}

通常可采用氩气雾化和等离子旋转电极两种 雾化方法制备 FGH95 合金粉末, 其化学成分分析和 测试的结果列于表 2 中(测量值区间)。氩气雾化制 粉(Argon atomization, AA)工艺是采用真空感应熔 炼母合金, 然后真空感应重熔, 并在氩雾化制粉设 备上制粉。而等离子旋转电极制粉(Plasma rotating electrode process, PREP)工艺是真空感应熔炼, 浇注 母合金棒, 然后在等离子旋转电极设备上雾化制粉。 通常氩气雾化粉末基本呈球形, 有成堆和块状黏结, 同时存在空心粉。等离子旋转电极雾化粉末呈球形, 表面光洁, 无粘粉现象。两种制粉方法雾化制粉原 理如图 1 所示, 在制粉过程中等离子弧将高速旋转 的棒料端面熔化, 在离心力的作用下, 熔化的液态 金属薄膜流向棒料端面的边缘, 由于表面张力的作 用, 液膜并不能立即从棒料端面甩出去, 而是在端 面形成了 “冠”, 金属熔体沿螺旋曲线不断地进入 “冠” 中，最后形成了 “露头”，当 “露头” 中金属 的质量增加到其离心力超过表面张力时, “露头” 便 从 “冠” 中飞射出去, 形成了小液滴。在惰性气体 中液滴以很高的速度冷却, 凝固成球形粉末颗粒 ${ }^{[3]}$ 。 分析受力, 可得到液滴形成的极限条件, 即 $m \omega^{2} D / 2 \geqslant \sigma \pi d_{1}$, 式中, $m$ 为液滴的质量, $D$ 为棒料 的直径, $\omega$ 为棒料旋转的角速度, $d_{1}$ 为液滴的直径, $\sigma$ 为金属熔滴表面张力。对于镍基高温合金，取 $\sigma=$ $1778 \mathrm{dyn} / \mathrm{cm}$, 粉末合金的平均密度 $\rho=7.77 \mathrm{~g} / \mathrm{cm}^{3}$ 代入关系式中可得出等离子旋转电极雾化制粉的粉 末尺寸理论值(直径 $d$ )表达式

$$
d=\frac{1.42 \times 10^{7}}{n} \frac{1}{\sqrt{D}}
$$

式中, $n$ 为电极棒转速。

表 2 FGH95 合金的化学成分(质量分数) $\%$

\begin{tabular}{cc||cc}
\hline 元素 & 测试值 & 元素 & 测试值 \\
\hline $\mathrm{Al}$ & $3.40 \sim 3.50$ & $\mathrm{Mn}$ & $<0.10$ \\
$\mathrm{~W}$ & $3.35 \sim 3.45$ & $\mathrm{Zr}$ & $0.04 \sim 0.07$ \\
$\mathrm{Nb}$ & $3.30 \sim 3.50$ & $\mathrm{Ta}$ & $<0.05$ \\
$\mathrm{Mo}$ & $3.30 \sim 3.48$ & $\mathrm{Si}$ & $0.050 \sim 0.110$ \\
$\mathrm{Ti}$ & $2.50 \sim 2.55$ & $\mathrm{P}$ & $<0.010$ \\
$\mathrm{Cr}$ & $12.85 \sim 13.07$ & $\mathrm{~S}$ & $0.002 \sim 0.003$ \\
$\mathrm{Fe}$ & $0.10 \sim 0.37$ & $\mathrm{C}$ & $0.045 \sim 0.067$ \\
$\mathrm{Co}$ & $7.95 \sim 8.02$ & $\mathrm{O}$ & $<0.005$ \\
$\mathrm{~B}$ & $0.011 \sim 0.014$ & $\mathrm{Ni}$ & 余量 \\
\hline
\end{tabular}

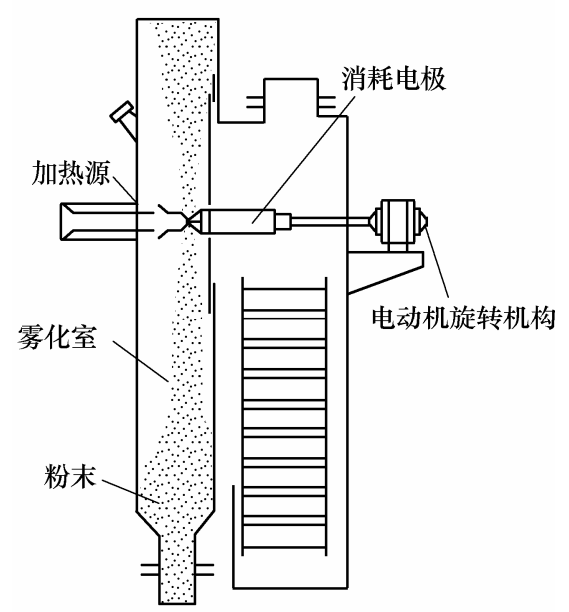

(a) PREP 制粉工艺

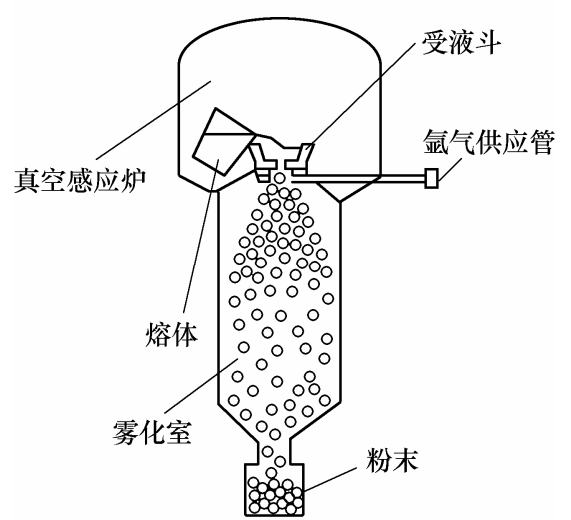

(b) AA 制粉工艺

图 1 FGH95 合金雾化制粉原理图

实际上，粉末颗粒的尺寸大小受棒料的振动等 的影响, 在理论值附近波动。由粉末粒度的理论表 达式可知，对于已知成分的合金其雾化粉末粒度与 电极棒直径和转速有直接关系。自 20 世纪 80 年代 开始，各国学者为了解决合金粉末中的非金属夹杂 对合金组织和性能影响的问题，开始降低所使用粉 末的粒度, 如使用粒度小于 $100 \mu \mathrm{m}$ 、小于 $75 \mu \mathrm{m}$ 和 小于 $50 \mu \mathrm{m}$ 等粉末，所使用粉末的粒度越小，则非 
金属夹杂的尺寸也越小, 其对合金性能的影响就越 低。对于氩气雾化制粉, 由于自身制粉特点决定获 得细粉相对容易, 目前最先进的氩气雾化制粉工艺 可保证粒度小于 $50 \mu \mathrm{m}$ 细粉收得率达到 $80 \%$ 以上。 而等离子旋转电极制粉工艺由于受到电极棒转速提 高所导致的噪声和振动等无法解决的问题限制, 目 前粒度小于 $100 \mu \mathrm{m}$ 粉末的收得率最高可达到 $60 \%$ 。 图 2 是试验所得的两种制粉方法粉末的粒度分布情 况 $^{[5-6]}$ 。

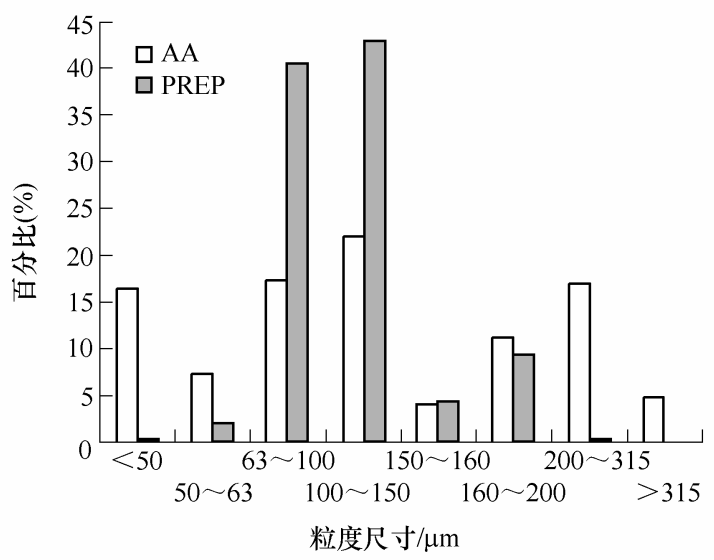

图 2 氩气雾化和等离子旋转电极雾化 FGH95 粉末的 粒度分布

分析 PREP 和 AA 两种制粉工艺, 由于前者需 要母合金棒料的真空熔炼和浇注、需要对棒料进行 表面和尺寸的精加工、雾化过程中还有 $8 \%$ 左右的 棒料头剩余, 因此工艺成本高, 同时设备相对复杂, 这些都将会导致合金粉末成本提高。AA 制粉相比 之下工艺成本低, 细粉收得率高, 但粉末的氧、氮 气体体积分数偏高, 易形成耐火材料和陶瓷等非金 属夹杂, 同时粉末的球形度差、空心率高, 正是由 于这些问题的存在, 我国目前 FGH95 合金粉末的主 导制粉工艺是 PREP 工艺, 如果能够克服 AA 法制 粉工艺的上述缺点, 采用 $\mathrm{AA}$ 法雾化制备 FGH95 合金粉末还是应大力推荐的。

\section{2 粉末的组织、形貌及质量}

PREP 和 AA 粉末的化学成分范围大都在表 2 所测试的范围内, 主要区别在 AA 粉末的 C、O、N 质量分数偏高, 这将会导致在随后热等静压密实成 形时易形成原始粉末颗粒边界(Previous particle boundary, PPB)等缺陷。FGH95 合金的两种形式粉 末组织和形貌上存在一定的区别, AA 粉末基本呈 球形, 但粉末有成堆和块状黏结, PREP 粉末球形 度好, 表面光亮洁净, 粉黏结少, 如图 3 所示, 两 种雾化制粉方法都属于金属液滴快速凝固成粉末， 因此粉末颗粒的内部和表面都呈铸态的枝晶组织,
只是冷却速度高, 枝晶间距小, 由于粉末中的枝晶 间距大都在 $5 \mu \mathrm{m}$ 以下，不会对合金的组织均匀性 产生影响, 这也正是粉末高温合金的优点之一。

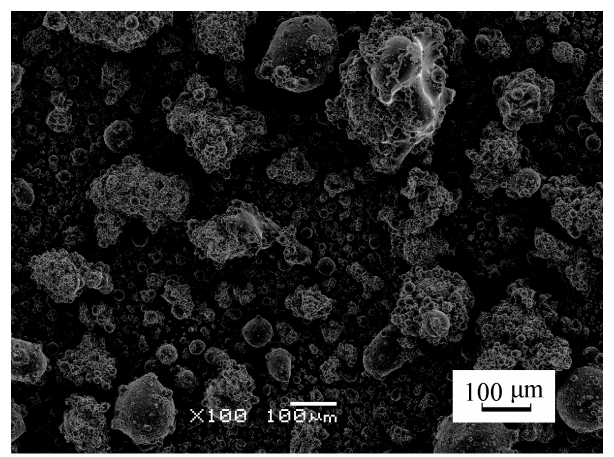

(a) AA 制粉工艺(低倍)

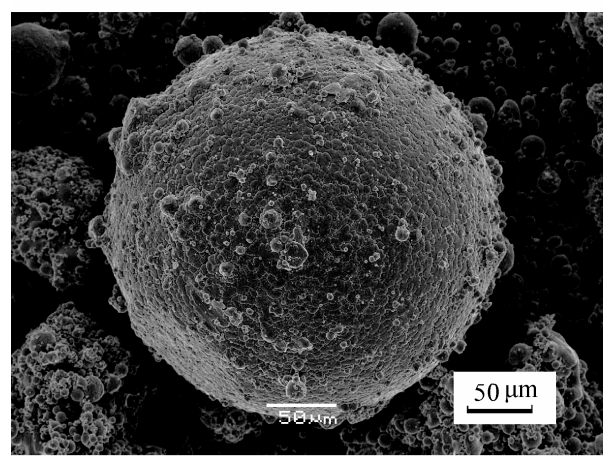

(b) AA 制粉工艺(高倍)

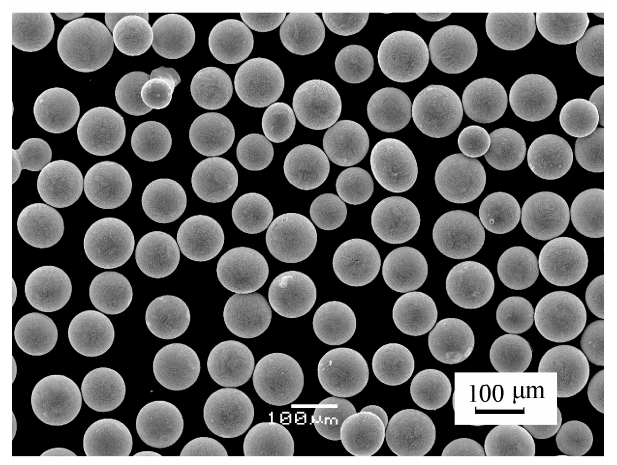

(c) PREP 制粉工艺(低倍)

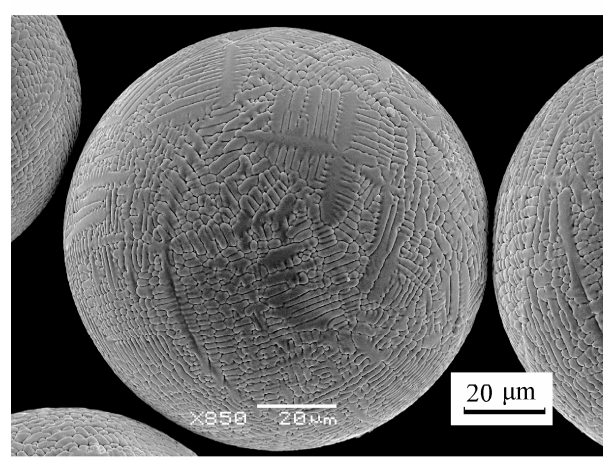

(d) PREP 制粉工艺(高倍)

图 3 FGH95 合金粉末的形貌和组织

用萃取的方法分析粉末颗粒内部的析出相表 明, 主要的析出相是 MC 型碳化物, 随着粉末颗粒 
尺寸(凝固速度)的不同, 碳化物的形状可能呈堆积 状、枝晶态和蜘网状等, 其大小(小于 $0.5 \mu \mathrm{m}$ )不会 对合金的凝固组织产生影响, 只是在热等静压成形 过程中碳化物在粉末颗粒边界的偏聚形成原始粉末 颗粒边界 PPB 才会影响合金的力学性能。

空心粉问题是粉末质量的一个重要问题, 因为 在压实成形过程中会形成不能完全压合的孔洞, 在 随后的热处理过程中就会形成热诱导孔洞, 影响合 金的性能。研究表明 AA 工艺 FGH95 粉末的空心率 高, 其形成原因主要有雾化介质气体的机械裹入、 气体的溶解析出及粉末颗粒的包覆等 ${ }^{[7]}$, 只有当粉 末粒度小于 $20 \mu \mathrm{m}$ 时空心粉末几乎可以忽略不计, 要真正消除 AA 粉空心所形成的热诱导空洞对性能 的影响, 必须采用热挤压工艺来破碎封闭的气体孔 洞。而 PREP 雾化粉末的空心率很低, 研究表明, 其主要与电极棒转速有直接关系, 电极棒转速提高, 空心粉尺寸变小, 数量增加。正是由于 PREP 粉末 空心率小、圆整度好、气体体积分数低, 因此其更 适用于直接热等静压成形。

\section{3 粉末的处理工艺}

由于镍基粉末高温合金主要用于制造航空发 动机关键部件等, 因此对粉末的质量要求非常高。 FGH95 合金粉末的高质量保证主要通过粉末处理 工艺来实现, 包括粉末粒度篮分、静电分离去除非 金属夹杂、粉末加热真空脱气等。其中非金属夹杂 的去除尤为重要, 直接影响到合金坏件能否符合质 量检测的要求、性能能否达到标准。对于 FGH95 高温合金粉末, 静电分离是去除非金属夹杂的主要 手段。对于俄式的三级静电分离设备, $12000 \mathrm{~V}$ 的 电晕电压、 $5000 \mathrm{~V}$ 的清理电压、60 r/min 的沉淀极 转速、 $100 \mathrm{~kg} / \mathrm{h}$ 的处理速度是分离夹杂的优化参数 组合, 在此参数下首次静电分离夹杂率可达 $75 \%$ 。 需要指出的是, 目前的静电分离工艺不是去除 FGH95 非金属夹杂的最优化工艺, 完全解决非金属 夹杂问题还必须改善熔炼工艺、细化粉末粒度、采 用大变形量(如热挤压等)成形工艺、预测夹杂物作 用下合金疲劳寿命等。

\section{2 成形工艺的研究}

成形工艺是镍基粉末高温合金的主要冶金工 艺之一, 对粉末高温合金的组织和性能具有决定作 用。对于 FGH95 粉末高温合金试验研究了直接热等 静压 (Hot isostatic pressing, HIP)、等温锻造 (Isothermal forging, IF)、热等静压+包套锻造(Hot isostatic pressing+Forging, HIP+F) 和热挤压 $($ Hot extrusion, HE)等几种成形工艺合金的组织和性能, 图 4 显示了 FGH95 合金不同成形工艺的组织。

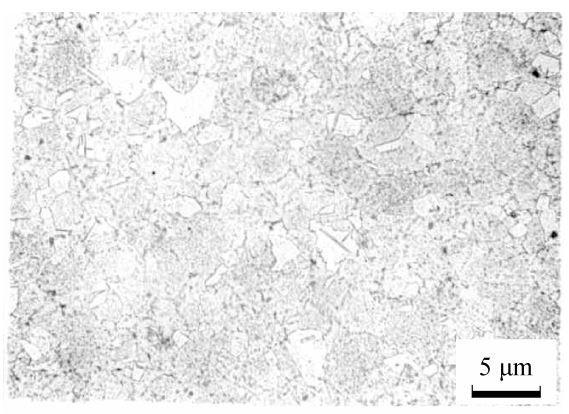

(a) 直接 HIP 成形

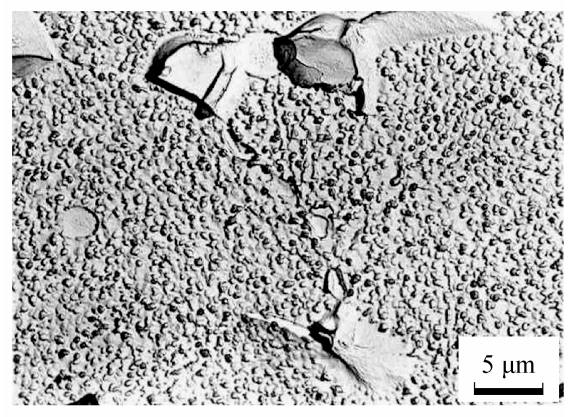

(b) (HIP+F)成形

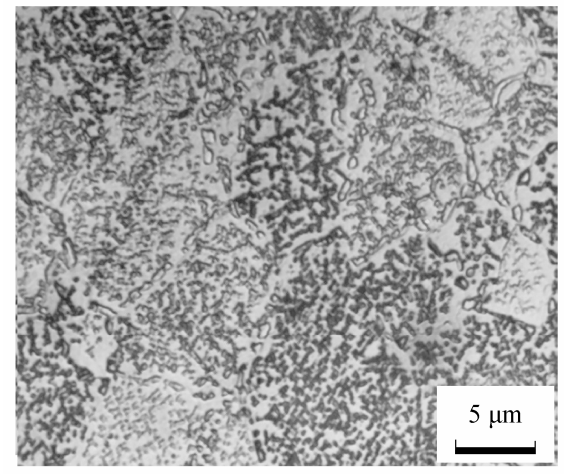

(c) 等温锻造

图 4 不同成形工艺 FGH95 合金的组织

研究表明, 粉末经热等静压成形后, 大部分颗 粒仍保持着雾化粉末的枝晶组织, 没有明显的塑性 变形, 晶粒度在 ASTM8 级以下, 残余枝晶面积最 高可达 $45 \%$, 大 $\gamma^{\prime}$ 相沿原始颗粒边界和晶界析出, 方形和细小球形 $\gamma^{\prime}$ 相分布在晶内, 另外少量的碳化 物主要分布在原始颗粒边界和晶界上。热等静压后 再加锻造成形, 大多数粉末颗粒发生形变再结晶, 随着变形量的增加, 残余枝晶数量减少, 一般残余 枝晶面积在 $10 \%$ 以下。锻造后 FGH95 合金的晶粒 度可达 ASTM9 级以上, 最高可达 ASTM12 级, 析 出相 $\gamma^{\prime}$ 和碳化物的分布与直接 HIP 合金相似。等温 锻造成形时, 由于应变速率低, 温度恒定, 因此组 织更加均匀, 甚至可产生超塑性变形, 晶粒更加细 小, 在高于 $\gamma^{\prime}$ 固溶线温度 $\left(1160{ }^{\circ} \mathrm{C}\right) \mathrm{HIP}$ 后锻造, 其 组织为未再结晶的温加工晶粒围绕着再结晶的项链 
状细晶粒, 如图 4c 所示, 这是粉末高温合金非常希 望得到的组织结构。因此 FGH95 合金的等温锻造工 艺应是一个重要的研究方向。FGH95 合金热挤压成 形时, 由于粉末颗粒受到强烈剪切变形, 形成完全 再结晶的等轴晶细晶组织, 枝晶和原始粉末颗粒边 界已完全不存在。晶粒度可达 ASTM12 级以上, 晶 粒尺寸与挤压比和挤压温度有关, 与原始粉末颗粒 尺寸关系不大。挤压比越大(通常可达 $12: 1$ ), 晶粒 尺寸越小, 挤压温度低于 $\gamma^{\prime}$ 相固溶线温度时, 晶粒 长大受 $\gamma^{\prime}$ 相阻碍, 得到细小的晶粒组织 ${ }^{[8]}$ 。

研究不同成形工艺 FGH95 合金的力学性能表 明, 热挤压合金综合性能最好, 其抗拉强度和塑性 均高于其他几种成形工艺。尤其是 $650{ }^{\circ} \mathrm{C}$ 持久强度 提高更为明显。热等静压+锻造的合金, 其室温和 $650{ }^{\circ} \mathrm{C}$ 抗拉强度及塑性也较直接热等静压合金有提 高。分析几种不同成形工艺的优缺点可知, 挤压和 等温锻成形工艺可以使 FGH95 合金获得良好的综 合力学性能, 但挤压的形状可变性小, 不能直接生 产锻坏形状成品, 等温锻造对模具材料要求高, 两 种成形工艺的设备复杂、成本高。直接 HIP FGH95 合金组织上存在大量残余枝晶和原始粉末颗粒边 界, 晶粒度受原始粉末颗粒尺寸影响较大, 力学性 能, 尤其是低周疲劳性能相比较低, 但工艺自身简 单、成本低, 产品形状可变性大。表 3 是不同工艺
参数下, 热等静压 FGH95 合金的力学性能, 结果表 明, 随着热等静压温度的升高, 抗拉强度、屈服强 度极限值降低、持久强度变化无规律, 比如 $1120{ }^{\circ} \mathrm{C}$ HIP 时, 其拉伸和屈服强度及塑性要好于 $1140{ }^{\circ} \mathrm{C}$, 但持久性能相比之下要低。从综合性能来看 1160 ${ }^{\circ} \mathrm{C}$ 的 HIP 温度对获得 FGH95 合金良好的性能是有 利的。1 $120{ }^{\circ} \mathrm{C}$ 相同温度下, 采用 $120 \mathrm{MPa}$ 和 150 $\mathrm{MPa}$ 的不同压力结果导致前者合金的塑性和持久 性能差, 出现缺口敏感性。这主要是因为不足的热 等静压压力, 导致粉末颗粒的塑性变形不充分, 因 而残余枝晶数量较大, 压后密实度不够, 这表明,

FGH95 合金的 HIP工艺符合粉末高温合金的高压力 有利于提高强度极限值和持久性能的 HIP 成形规 律。笔者采用直接 HIP 成形工艺已成功研制了 FGH95 合金的涡轮挡板制件。同时由于目前还不具 备大型等温锻造和热挤压设备, 因此研制大尺寸浴 轮盘件采用热等静压加包套锻造成形。图 5 是目前 主要研制的两种 FGH95 合金制件的成形工艺示意 图。从粉末高温合金发展近 30 多年的历史来看, 高 成本一直是阻碍其广泛应用的主要问题之一，而采 用低成本的成形工艺(如直接热等静压)可以大幅度 降低成本, 因此 FGH95 合金的直接 HIP 成形工艺 将是今后研究的一个重点 ${ }^{[9-10]}$ 。

表 3 热等静压 FGH95 合金的性能

\begin{tabular}{|c|c|c|c|c|c|c|c|c|c|c|c|c|}
\hline \multirow{4}{*}{$\begin{array}{l}\text { 热等静 } \\
\text { 压工艺 }\end{array}$} & \multicolumn{4}{|c|}{ 室温拉伸性能 } & \multicolumn{4}{|c|}{$650^{\circ} \mathrm{C}$ 拉伸性能 } & \multicolumn{4}{|c|}{ 持久性能, $650{ }^{\circ} \mathrm{C}, 1034 \mathrm{MPa}$} \\
\hline & \multirow{3}{*}{$\begin{array}{c}\text { 抗拉强度 } \\
/ \mathrm{MPa}\end{array}$} & \multirow{3}{*}{$\begin{array}{c}\text { 屈服强度 } \\
/ \mathrm{MPa}\end{array}$} & \multirow{3}{*}{$\begin{array}{c}\text { 伸长率 } \\
(\%)\end{array}$} & \multirow{3}{*}{$\begin{array}{c}\text { 断面收缩率 } \\
(\%)\end{array}$} & \multirow{3}{*}{$\begin{array}{c}\text { 抗拉强度 } \\
/ \mathrm{MPa}\end{array}$} & \multirow{3}{*}{$\begin{array}{c}\text { 屈服强度 } \\
\text { /MPa }\end{array}$} & \multirow{3}{*}{$\begin{array}{c}\text { 伸长率 } \\
(\%)\end{array}$} & \multirow{3}{*}{$\begin{array}{c}\text { 断面收缩率 } \\
(\%)\end{array}$} & \multirow{2}{*}{\multicolumn{3}{|c|}{ 光滑持久 }} & \multirow{3}{*}{$\begin{array}{l}\text { 缺口持久 } \\
r=0.2 \mathrm{~mm} \\
\text { 持久寿命 } \mathrm{h}\end{array}$} \\
\hline & & & & & & & & & & & & \\
\hline & & & & & & & & & 持久寿命 $/ \mathrm{h}$ & 伸长率(\%) & 断面收缩率(\%) & \\
\hline \multirow{2}{*}{$A^{(1)}$} & 1645 & 1296 & 11.3 & 14.6 & 1472 & 1136 & 7.0 & 9.0 & 181.17 & 3.6 & 3.56 & 7.17 \\
\hline & 1579 & 1314 & 8.0 & 11.7 & 1410 & 1139 & 5.2 & 10.7 & 179.00 & 2.64 & 2.79 & 0.58 \\
\hline \multirow{2}{*}{$\mathrm{B}^{(2)}$} & 1623 & 1329 & 10.6 & 11.2 & 1583 & 1243 & 7.0 & 10.5 & 306.55 & 1.20 & 4.15 & $>306.58$ \\
\hline & 1644 & 1319 & 12.4 & 12.7 & 1576 & 1243 & 8.0 & 9.7 & 298.00 & 1.60 & 3.96 & $>298.00$ \\
\hline \multirow{2}{*}{$\mathrm{C}^{(3)}$} & 1642 & 1347 & 9.6 & 11.6 & 1552 & 1238 & 7.0 & 10.5 & 422.50 & 2.0 & 6.30 & $>422.50$ \\
\hline & 1569 & 1301 & 9.4 & 10.7 & 1552 & 1243 & 8.0 & 9.7 & 372.75 & 1.60 & 4.15 & $>372.75$ \\
\hline \multirow{2}{*}{$\mathrm{D}^{(4)}$} & 1683 & 1310 & 12.4 & 14.0 & 1557 & 1253 & 8.0 & 12.0 & 260.08 & 3.20 & 2.79 & $>260.08$ \\
\hline & 1562 & 1340 & 12.0 & 12.1 & 1538 & 1243 & 8.0 & 12.3 & 329.58 & 3.60 & 4.34 & $>329.55$ \\
\hline \multirow{2}{*}{$E^{(5)}$} & 1559 & 1299 & 10.0 & 12.1 & 1567 & 1228 & 10.4 & 12.0 & 397.83 & 2.0 & 4.34 & $>397.83$ \\
\hline & 1590 & 1278 & 10.0 & 12.2 & 1503 & 1213 & 7.2 & 8.6 & 465.67 & 4.0 & 3.37 & $>465.67$ \\
\hline 标准(A) & 1590 & 1240 & 10 & 12 & 1430 & 1150 & 8 & 10 & $>50$ & $>3$ & - & $\begin{array}{c}\text { 缺口持久寿 } \\
\text { 命 }>\text { 光滑持 } \\
\text { 久寿命 }\end{array}$ \\
\hline
\end{tabular}

注: (1) A: $1120{ }^{\circ} \mathrm{C} 、 120 \mathrm{MPa} \times 3 \mathrm{~h}$; (2) B: $1120{ }^{\circ} \mathrm{C} 、 150 \mathrm{MPa} \times 3 \mathrm{~h}$; (3) $\mathrm{C}: 1140{ }^{\circ} \mathrm{C} 、 150 \mathrm{MPa} \times 3 \mathrm{~h}$; (4) $\mathrm{D}: 1160{ }^{\circ} \mathrm{C} 、 150 \mathrm{MPa} \times 3 \mathrm{~h}$; (5) $\mathrm{E}: 1180{ }^{\circ} \mathrm{C}$, $150 \mathrm{MPa} \times 3 \mathrm{~h}$ 


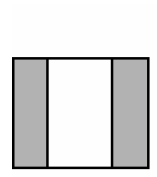

装套、封焊

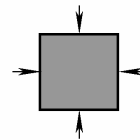

热等静压

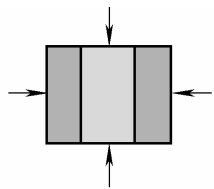

HIP成形

(a) 涡轮

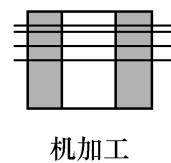

机加工

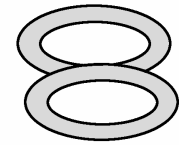

涡轮挡板

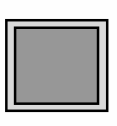

包套

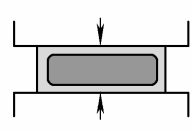

镦粗

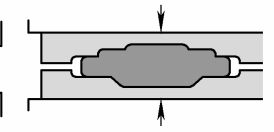

模锻 (b) 涡轮盘的成形工艺

图 5 FGH95 合金浴轮盘和浴轮挡板成形工艺示意图

\section{FGH95 合金的热处理工艺}

\subsection{FGH95 合金热处理工艺参数的优化}

对于镍基粉末高温合金热处理工艺的合理性 直接关系到最终材料的组织和性能, 主要体现为 $\gamma^{\prime}$ 相数量及尺寸分布以及晶粒组织的变化。热处理工 艺参数主要包括固溶温度、保温时间、淬火介质和 温度及时效制度等。其中时效制度主要是保证更多 的细小 $\gamma^{\prime}$ 相析出, 同时使碳化物的分布更加合理, 组织更加稳定, 从而进一步提高性能。时效制度的 两个主要参数是时效温度和时效的次数, 一般二次 时效后, 细小 $\gamma^{\prime}$ 相数量将增加。对于 FGH95 粉末高 温合金, 研究认为有几种时效制度较适宜。如: (1) $870{ }^{\circ} \mathrm{C} / 1 \mathrm{~h}$ 空冷 $+650{ }^{\circ} \mathrm{C} / 24 \mathrm{~h}$ 空冷; (2) $760{ }^{\circ} \mathrm{C} / 16$ $\mathrm{h}$ 空冷或 $870{ }^{\circ} \mathrm{C} / 1 \mathrm{~h}$ 空冷; (3) $650{ }^{\circ} \mathrm{C} / 24 \mathrm{~h}$ 空冷 等, 研究表明(2)、(3)两种时效制度可以改善晶界碳 化物的形态和合理分布, 从而提高合金的持久性能, (1)时效制度可以保证细小 $\gamma^{\prime}$ 相的充分析出, 而且碳 化物的析出和分布更加稳定化, 因此其拉伸和屈服 强度较好, 蠕变性能也得以改善, 持久性能可以满 足要求, 所以对于 FGH95 合金时效制度通常采用具 有二级时效的(1)制度。

对于镍基粉末高温合金的热处理工艺来说，固 溶处理是为了溶解基体内碳化物、 $\gamma^{\prime}$ 相等, 从而得 到均匀的固溶体, 以便时效时均匀地析出 $\gamma^{\prime}$ 相, 另 外通过固溶处理可以获得适宜的晶粒度, 以便提高 合金的强度或高温蠕变性能等。固溶温度的高低通 常是以 $\gamma^{\prime}$ 相溶解温度为基准的。高于 $\gamma^{\prime}$ 相的溶解温 度, 阻碍晶粒长大的晶界大 $\gamma^{\prime}$ 相及一些碳化物溶解 或转化, 因此晶粒长大, 从而提高合金的高温蠕变 和持久性能, 而如果在 $\gamma^{\prime}$ 相溶解温度以下处理, 则 晶粒度保持锻造变形后的状态, 较细小, 因而抗拉 强度、屈服强度及疲劳强度等性能较好。FGH95 通 常采用在 $\gamma^{\prime}$ 溶解温度 $\left(1160{ }^{\circ} \mathrm{C}\right)$ 以下进行固溶处理,
从而使 FGH95 合金在 $650{ }^{\circ} \mathrm{C}$ 高温下仍具有较高的 拉伸和屈服强度。对于粉末高温合金热处理工艺所 采用的冷却方式基本上有如下几种：炉冷、空冷、

气淬、盐淬、油淬、水冷等。通常美国常采用油淬 冷却，而俄罗斯采用空冷或风冷及炉冷等。我国在 大量试验研究的基础上确定盐淬作为 FGH95 粉末 高温合金热处理工艺淬火介质的首选。

表 4 和表 5 是 FGH95 合金不同固溶温度 $600{ }^{\circ} \mathrm{C}$ 盐淬后的性能数据。根据研究结果和经验, 使用盐 淬作为淬火冷却方式虽然可以避免油淬时冷速过大 所导致的淬裂问题和解决空冷、炉冷等冷速过低所 导致的强度指标不能满足要求的缺点等, 但盐浴炉 所带来的对环境和设备的腐蚀及操作过程的复杂性 等, 都阻碍了这一工艺方法在连续运转的工业化生 产条件下的应用。因此, 深入研究 FGH95 粉末高温 合金的适宜的淬火介质，改进淬火工艺都将是今后 热处理工艺的研究重点。

表 4 FGH95 合金盐淬热处理的拉伸性能

\begin{tabular}{|c|c|c|c|c|c|}
\hline $\begin{array}{c}\text { 固溶 } \\
\text { 温度 } /{ }^{\circ} \mathrm{C} \\
\end{array}$ & $\begin{array}{c}\text { 拉伸 } \\
\text { 温度/ } /{ }^{\circ} \mathrm{C}\end{array}$ & $\begin{array}{c}\text { 抗拉强度 } \\
\text { /MPa }\end{array}$ & $\begin{array}{c}\text { 屈服强度 } \\
/ \mathrm{MPa}\end{array}$ & 伸长率(\%) & $\begin{array}{c}\text { 断面收缩 } \\
\text { 率(\%) }\end{array}$ \\
\hline \multirow{4}{*}{1120} & \multirow{2}{*}{20} & 1598 & 1298 & 14.2 & 17.4 \\
\hline & & 1612 & 1236 & 15.8 & 14.7 \\
\hline & \multirow{2}{*}{650} & 1515 & 1149 & 16.4 & 17.4 \\
\hline & & 1487 & 1141 & 12.3 & 13.7 \\
\hline \multirow{4}{*}{1140} & \multirow{2}{*}{20} & 1618 & 1284 & 13.4 & 14.3 \\
\hline & & 1630 & 1280 & 15.3 & 17.5 \\
\hline & \multirow{2}{*}{650} & 1531 & 1176 & 13.2 & 14.5 \\
\hline & & 1543 & 1171 & 13.9 & 14.8 \\
\hline \multirow{4}{*}{1160} & \multirow{2}{*}{20} & 1649 & 1321 & 15.7 & 15.7 \\
\hline & & 1657 & 1336 & 13.6 & 12.9 \\
\hline & \multirow{2}{*}{650} & 1559 & 1220 & 9.4 & 12.9 \\
\hline & & 1515 & 1200 & 8.5 & 13.3 \\
\hline \multirow{4}{*}{1180} & \multirow{2}{*}{20} & 1641 & 1339 & 12.2 & 12.1 \\
\hline & & 1654 & 1342 & 13.6 & 14.5 \\
\hline & \multirow{2}{*}{650} & 1556 & 1217 & 10.0 & 13.7 \\
\hline & & 1532 & 1230 & 7.2 & 6.5 \\
\hline
\end{tabular}

表 5 FGH95 合金盐淬热处理的持久性能

\begin{tabular}{|c|c|c|c|c|c|}
\hline \multirow{2}{*}{$\begin{array}{c}\text { 固溶 } \\
\text { 温度 } /{ }^{\circ} \mathrm{C}\end{array}$} & \multirow{2}{*}{$\begin{array}{c}\text { 温度 } \\
/{ }^{\circ} \mathrm{C}\end{array}$} & \multirow{2}{*}{$\begin{array}{l}\text { 应力 } \\
/ \mathrm{MPa}\end{array}$} & \multicolumn{2}{|c|}{ 光滑持久 } & \multirow{2}{*}{$\begin{array}{c}\text { 缺口持久 }{ }^{(1)} \\
\text { 持久 } \\
\text { 寿命 } / \mathrm{h}\end{array}$} \\
\hline & & & $\begin{array}{l}\text { 持久 } \\
\text { 寿命/h }\end{array}$ & $\begin{array}{c}\text { 断面收缩率 } \\
(\%)\end{array}$ & \\
\hline \multirow{2}{*}{1120} & \multirow{2}{*}{650} & \multirow{2}{*}{1034} & 401.08 & 2.84 & 272.25 \\
\hline & & & 383.50 & 1.51 & $>802.50$ \\
\hline \multirow{2}{*}{1140} & \multirow{2}{*}{650} & \multirow{2}{*}{1034} & 382.00 & 3.31 & 537.50 \\
\hline & & & 526.33 & 3.6 & $>802.50$ \\
\hline \multirow{2}{*}{1160} & \multirow{2}{*}{650} & \multirow{2}{*}{1034} & 516.33 & 1.51 & 131.58 \\
\hline & & & 460.67 & 1.69 & 168.25 \\
\hline \multirow{2}{*}{1180} & \multirow{2}{*}{650} & \multirow{2}{*}{1034} & 506.43 & 2.22 & 4.50 \\
\hline & & & 426.33 & 1.78 & 55.50 \\
\hline
\end{tabular}

注: (1) 缺口半径 $r=0.2 \mathrm{~mm}$ 。 


\section{2 提高 FGH95 合金抗疲劳裂纹扩展能力的方法}

FGH95 粉末高温合金虽然在 $650{ }^{\circ} \mathrm{C}$ 具有较高 的抗拉强度, 但其抗疲劳裂纹扩展能力较低。目前 为提高航空发动机的安全可靠性, 要求发动机的关 键部件材料必须符合损伤容限设计原理, 即要求合 金具有较高的抗裂纹扩展能力 ${ }^{[11]}$ 。对于镍基粉末高 温合金来说, 通常粗晶粒和细小 $\gamma^{\prime}$ 相阻碍裂纹扩展。 研究表明, 改变固溶温度和固溶冷却速度可以在一 定程度上改变 FGH95 合金的组织结构, 提高固溶温 度和减低冷却速度就可以增大晶粒尺寸和溶解晶界 大 $\gamma^{\prime}$ 相、析出小尺寸 $\gamma^{\prime}$ 相。表 6、表 7 和图 6 显示 了采用缓冷热处理工艺 FGH95 合金的裂纹扩展速 率的改善和性能变化。结果表明, 采用 $\mathrm{B}$ 制度的高 固溶缓冷处理后, 合金的疲劳裂纹扩展速度明显降 低, 蠕变状态下裂纹扩展的应力强度因子 $\Delta K$ 升高, 这是因为高固溶缓冷处理后合金的晶粒长大，大尺 寸 $\gamma^{\prime}$ 相溶解, 同时产生部分弯曲晶界, 因此合金疲 劳裂纹扩展速率明显降低, 持久和塑性显著提高, 但强度水平有所下降, 这符合损伤容限设计原理。

表 6 高固溶缓冷热处理 FGH95 合金的性能

\begin{tabular}{|c|c|c|c|c|c|}
\hline $\begin{array}{c}\text { 热处理 } \\
\text { 制度 }\end{array}$ & $\begin{array}{c}\text { 拉伸 } \\
\text { 温度/ }\end{array}$ & $\begin{array}{c}\text { 抗拉强度 } \\
/ \mathrm{MPa} \\
\end{array}$ & $\begin{array}{c}\text { 屈服强度 } \\
\mathrm{MPa} \\
\end{array}$ & $\begin{array}{c}\text { 伸长率 } \\
(\%)\end{array}$ & $\begin{array}{l}\text { 断面收 } \\
\text { 缩率 }(\%)\end{array}$ \\
\hline \multirow{4}{*}{$\mathrm{A}^{(1)}$} & \multirow{2}{*}{20} & 1618 & 1161 & 19.44 & 23.0 \\
\hline & & 1650 & 1170 & 19.5 & 27.0 \\
\hline & \multirow{2}{*}{650} & 1467 & 1100 & 14.4 & 13.9 \\
\hline & & 1462 & 1104 & 12.8 & 12.8 \\
\hline \multirow{4}{*}{$\mathrm{B}^{(2)}$} & \multirow{2}{*}{20} & 1510 & 1095 & 18.5 & 25.5 \\
\hline & & 1485 & 1080 & 17.1 & 24.0 \\
\hline & \multirow{2}{*}{650} & 1405 & 976 & 20.2 & 21.5 \\
\hline & & 1400 & 973 & 21.9 & 23.3 \\
\hline
\end{tabular}

注: (1) A: $1120{ }^{\circ} \mathrm{C} / 1 \mathrm{~h}\left(1.2{ }^{\circ} \mathrm{C} / \mathrm{s}\right.$ 冷至 $) 600{ }^{\circ} \mathrm{C} / \mathrm{AC}+870{ }^{\circ} \mathrm{C} / 1 \mathrm{~h} / \mathrm{AC}+650$ ${ }^{\circ} \mathrm{C} / 24 \mathrm{~h} / \mathrm{AC}$; (2) B: $1160{ }^{\circ} \mathrm{C}\left(4{ }^{\circ} \mathrm{C} / \mathrm{min}\right.$ 缓冷至 $) 1120{ }^{\circ} \mathrm{C} / 1 \mathrm{~h}\left(1.2{ }^{\circ} \mathrm{C} / \mathrm{s}\right.$ 冷 至) $600{ }^{\circ} \mathrm{C} / \mathrm{AC}+870{ }^{\circ} \mathrm{C} / 1 \mathrm{~h} / \mathrm{AC}+650{ }^{\circ} \mathrm{C} / 24 \mathrm{~h} / \mathrm{A}$ 。

表 7 高固溶缓冷热处理 FGH95 合金的持久性能

\begin{tabular}{|c|c|c|c|c|c|c|}
\hline \multirow{2}{*}{$\begin{array}{c}\text { 热处理 } \\
\text { 制度 }\end{array}$} & \multirow{2}{*}{$\begin{array}{c}\text { 温度 } \\
/{ }^{\circ} \mathrm{C}\end{array}$} & \multirow{2}{*}{$\begin{array}{l}\text { 应力 } \\
/ \mathrm{MPa}\end{array}$} & \multicolumn{3}{|c|}{ 光滑持久 } & \multirow{2}{*}{$\begin{array}{l}\text { 缺口持久 } \\
r=0.2 \mathrm{~mm}\end{array}$} \\
\hline & & & $\begin{array}{c}\text { 持久寿 } \\
\text { 命 } / \mathrm{h}\end{array}$ & $\begin{array}{l}\text { 伸长 } \\
\text { 率(\%) }\end{array}$ & $\begin{array}{l}\text { 断面收 } \\
\text { 缩率(\%) }\end{array}$ & \\
\hline \multirow{2}{*}{$\mathrm{A}^{(1)}$} & \multirow{2}{*}{650} & 1 & 22 & 1.44 & 2.34 & 10 \\
\hline & & 034 & 27 & 1.84 & 2.34 & 8 \\
\hline \multirow{2}{*}{$\mathrm{B}^{(2)}$} & \multirow{2}{*}{650} & 1 & 69 & 10.0 & 7.03 & $>100$ \\
\hline & & 034 & 57 & 8.8 & 5.50 & $>100$ \\
\hline
\end{tabular}

注: (1) A: $1120{ }^{\circ} \mathrm{C} / 1 \mathrm{~h}\left(1.2{ }^{\circ} \mathrm{C} / \mathrm{s}\right.$ 冷至 $) 600{ }^{\circ} \mathrm{C} / \mathrm{AC}+870{ }^{\circ} \mathrm{C} / 1 \mathrm{~h} / \mathrm{AC}+650$ ${ }^{\circ} \mathrm{C} / 24 \mathrm{~h} / \mathrm{AC}$; (2)B: $1160{ }^{\circ} \mathrm{C}\left(4{ }^{\circ} \mathrm{C} / \mathrm{min}\right.$ 缓冷至 $) 1120{ }^{\circ} \mathrm{C} / 1 \mathrm{~h}\left(1.2{ }^{\circ} \mathrm{C} / \mathrm{s}\right.$ 冷至 $) 600$ ${ }^{\circ} \mathrm{C} / \mathrm{AC}+870{ }^{\circ} \mathrm{C} / 1 \mathrm{~h} / \mathrm{AC}+650{ }^{\circ} \mathrm{C} / 24 \mathrm{~h} / \mathrm{A}$ 。

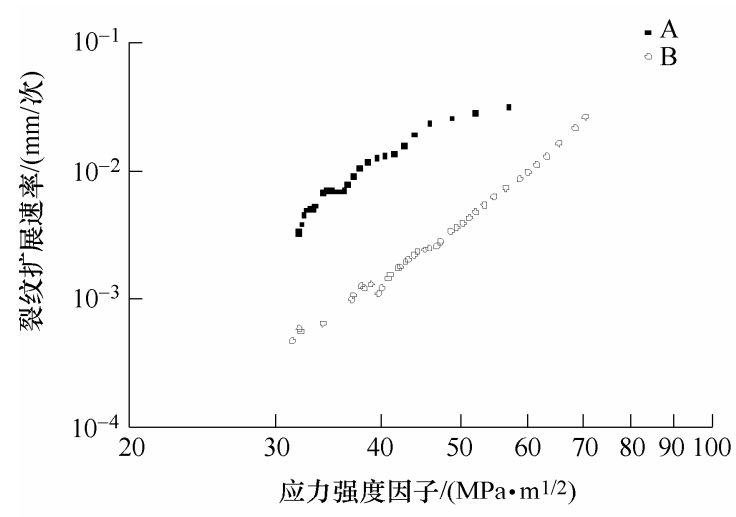

(a) 保载 $5 \mathrm{~s}$

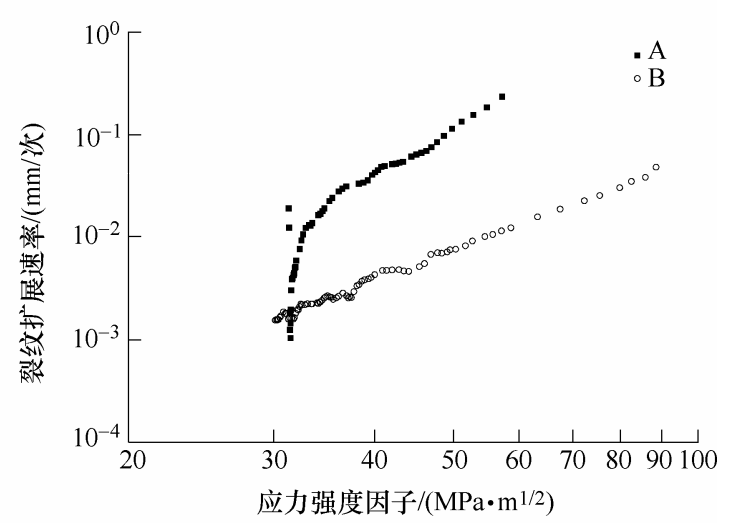

(b) 保载 $90 \mathrm{~s}$

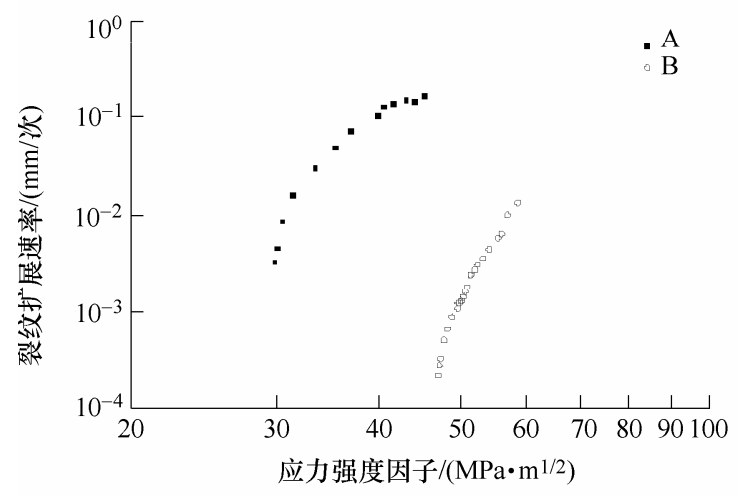

(c) 纯蠕变

图 6 FGH95 合金的特殊热处理后的裂纹扩展速率

\section{4 结论}

FGH95 镍基粉末高温合金是我国自主研制的 第一个粉末高温合金牌号, 在研制过程中引进并建 成了以等离子旋转电极设备为主的粉末高温合金生 产线。经过 20 多年的发展，在母合金熔炼工艺、雾 化制粉工艺、粉末处理工艺、成形工艺、热处理工 艺、质量控制及组织性能数据积累等方面都开展了 深入广泛的研究，取得了显著的成绩。目前合金 FGH95 合金在组织和性能方面已基本达到了标准 的要求, 可以作为先进航空发动机涡轮盘的制造材 
料。需要解决的问题是减少合金中非金属夹杂的数 量和尺寸。今后研究的重点是提高 FGH95 合金的综 合性能指标, 采用直接 HIP 成形工艺研制 FGH95 粉末浴轮盘, 同时研制以 FGH95 合金为基的双性能 粉末浴轮盘。

\section{参 考 文 献}

[1] 国为民, 宋普生, 吴剑涛, 等. 粉末高温合金的研制和 展望[J]. 粉末冶金工业, 1999, 9(2)：9-16.

GUO Weimin, SONG Pusheng, WU Jiantao, et al. Developments and prospects of powder metallurgy superalloys[J]. Powder Metallurgy Industry, 1999, 9(2): 9-16.

[2] 张义文, 上官永恒. 粉末高温合金的研究与发展 [J]. 粉 末治金工业，2004(6)：30-43.

ZHANG Yiwen, SHANGGUAN Yongheng. Research and development in $\mathrm{P} / \mathrm{M}$ superalloy[J]. Powder Metallurgy Industry, 2004(6): 30-43.

[3] 邹金文, 汪武祥. 粉末高温合金研究进展与应用[J]. 航 空材料学报, 2006(3): 243-250.

ZOU Jinwen, WANG Wuxiang. Development and application of P/M superalloy[J]. Journal of Aeronautical Materials, 2006(3): 243-250.

[4] 俞克兰, 周光垓. 盘件用粉末高温合金的发展与应用 $[\mathrm{C}] /$ FGH95 粉末高温合金论文集, 北京: 航空材料研 究所, 1990, 7: 1-8.

YU Kelan, ZHOU Guanggai. Development and application of P/M superalloy[C]/Proceeding for FGH95 PM Superalloy, Beijing: Beijing Institute of Aeronautical Materials, 1990, 7: 1-8.

[5] 张义文, 张莹, 陈生大, 等. PREP 制取高温合金粉末 特点 [J]. 粉末冶金技术, 2001，19(1): 12-15.

ZHAG Yiwen, ZHANG Ying, CHEN Shengda, et al. Characteristics of prep in preparing superalloy powder[J]. Powder Metallurgy Technology, 2001, 19(1): 12-15.

[6] 张荣. 用两种方法制造的镍基高温合金粉末 [J]. 粉末 治金技术，2001，18(增刊)：1-5.
ZHANG Ying. PM nickel based superalloys with two fabricationprocessing[J]. Powder Metallurgy Technology, 2001, 18(Suppl.): 1-5.

[7] 杜晓梅. 氩雾化 Rene95 和 FGH95 空心粉末研究 $[\mathrm{C}] / \mathrm{FGH} 95$ 粉末高温合金论文集, 北京: 航空材料研 究所, 1990, 7: 69-74.

DU Xiaomei. Investigation on AA hollow powder of rene 95 and FGH95 alloy[C]/Proceeding for FGH95 PM Superalloy, Beijing: Beijing Institute of Aeronautical Materials, 1990, 7: 69-74.

[8] 杨士仲, 李力. 粉末冶金高温合金 $[\mathrm{C}] /$ 高温合金四十年 文集, 北京: 钢铁研究总院, 1996: 65-72.

YANG Shizhong, LI Li. PM superalloy[C]//Proceedings for the Development of Superalloy for 40 Years, Beijing: CISRI, 1996: 65-72.

[9] 国为民，张风戈，冯涤，等. 不同生产工艺对 FGH95 粉末高温合金组织和性能的影响 [J]. 粉末治金工业, 2001(5): 7-12.

GUO Weimin, ZHANG Fengge, FENG Di, et al. Effects of producing process on microstructure and properties of FGH95 P/M superalloy[J]. Powder Metallurgy Industry, 2001(5): 7-12.

[10] 张义文. 热等静压技术新进展 [J]. 粉末治金工业, 2009(4): 32-39.

ZHANG Yiwen. Development in HIP technology[J]. Powder Metallurgy Industry, 2009(4): 32-39.

[11] 胡本芙, 田高峰, 贾成厂, 等. 双性能粉末高温合金浴 轮盘的研究进展[J]. 航空材料学报, 2007(8): 80-84.

HU Benfu, TIAN Gaofeng, JIA Chengchang, et al. Development in double-properties turbine disk of $\mathrm{P} / \mathrm{M}$ superalloy[J]. Journal of Aeronautical Materials, 2007(8): 80-84.

作者简介: 国为民, 男, 1965 年出生, 博士, 教授。主要从事高温合金 材料和工艺研究。

E-mail: fmguowm@sina.com 